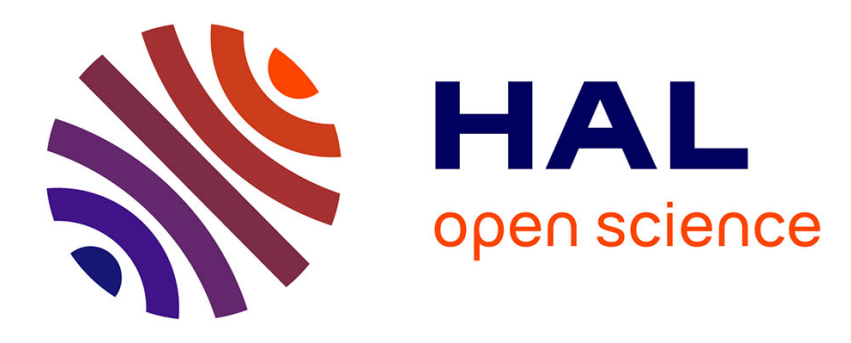

\title{
Tendances observées sur les régimes hydrologiques de l'arc Alpin
}

A. Bard, Benjamin Renard, M. Lang

\section{To cite this version:}

A. Bard, Benjamin Renard, M. Lang. Tendances observées sur les régimes hydrologiques de l'arc Alpin. La Houille Blanche - Revue internationale de l'eau, 2012, 1, p. 38 - p. 43. 10.1051/lhb/2012006 . hal-00727810

\section{HAL Id: hal-00727810 https://hal.science/hal-00727810}

Submitted on 4 Sep 2012

HAL is a multi-disciplinary open access archive for the deposit and dissemination of scientific research documents, whether they are published or not. The documents may come from teaching and research institutions in France or abroad, or from public or private research centers.
L'archive ouverte pluridisciplinaire HAL, est destinée au dépôt et à la diffusion de documents scientifiques de niveau recherche, publiés ou non, émanant des établissements d'enseignement et de recherche français ou étrangers, des laboratoires publics ou privés. 


\title{
Tendances observées sur les régimes hydrologiques de l’Arc Alpin
}

\author{
Antoine BARD ${ }^{1}$, Benjamin RENARD ${ }^{2}$, Michel $\mathrm{LANG}^{3}$
}

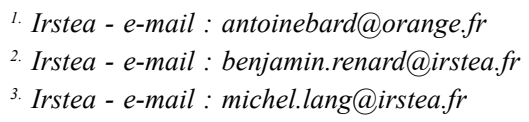

RÉSUMÉ. - Depuis 2008 le projet Européen AdaptAlp a pour vocation d'étudier les impacts du changement climatique dans les Alpes et d'évaluer les stratégies d'adaptation pour y faire face. Un des objectifs de ce projet est de faire un état des lieux des changements passés dans les régimes hydrologiques des rivières alpines.

Un nouveau jeu de données de 177 séries de débit journalier a été ainsi collecté, afin de couvrir l'ensemble de l'Arc Alpin. Ces séries représentent des bassins versants de montagne exempts d'influences anthropiques majeures et couvrent au moins 40 ans de mesures. L'ensemble des rivières étudiées balaie tout le spectre de comportements hydrologiques typiques des régions de montagne : des rivières purement glaciaires et nivales aux régimes mixtes, soumis aux influences conjuguées de précipitations liquides et solides.

De nombreux indices hydrologiques sont définis afin de caractériser les basses, moyennes et hautes eaux, en termes d'intensité et de saisonnalité. Une analyse statistique locale permet de tester la stationnarité et d'évaluer les tendances pour chaque site. Puis la significativité de ces changements à l'échelle régionale est estimée.

Les résultats montrent à l'échelle des Alpes des changements significatifs pour les étiages hivernaux qui tendent à être moins sévères. Les crues nivales présentent des changements de saisonnalité marqués : onde de fonte plus précoce et de durée allongée. Cependant aucune évolution nette dans leur intensité n'a été détectée à l'échelle globale.

Mots clefs : hydrologie, Alpes, fonte, détection de tendances, régime

\section{Observed Trends in the hydrologic regime of Alpine catchments}

\begin{abstract}
A European project, AdaptAlp, has been set up since 2008 to study climate change impacts in the Alps and to assess adaptation strategies. One of the objectives of this project is to study past changes in the hydrologic regime of Alpine rivers. This presentation shows the main results of a trend analysis.

A new dataset of 177 runoff time series has been collected over the alpine space. These series cover at least forty years of daily record and are related to undisturbed catchments. This dataset covers the whole spectrum of hydrological regimes existing in the Alps (from glacier- to mixed rainfall/snowmelt regimes).

In a second step, a set of hydrologic indices has been defined to characterize the hydrologic regime in terms of low, medium and high flows. In particular, these indices describe the drought severity and seasonality, the volume and timing of snowmelt flows, flood intensity and seasonality.

At-site statistical tests are applied for each hydrologic indice, and regional field significance is tested. Consistent trends affecting the timing of snowmelt-dominated flows are found. Winter droughts tend to be less severe (in terms of volume and duration), and spring floods seasonality appears to be shifted earlier in the season.
\end{abstract}

Key words : hydrology, Alps, snowmelt, trend detection, regime

\section{INTRODUCTION}

Les régions de montagne fournissent l'eau potable à environ la moitié de la population mondiale. Le Rhin, le Pô et le Rhône prennent leurs sources dans les Alpes, et celles-ci fournissent en moyenne entre 30 et $50 \%$ de leur débit total [Viviroli et Weingartner, 2004]. Alors que le réchauffement climatique est aujourd'hui considéré sans équivoque par la communauté scientifique [Bernstein et al., 2007 ; Puget et al., 2010], les régions de montagne devraient impactées du fait de l'augmentation attendue des températures. Néanmoins nombre d'études de stationnarité (entre autres Kundzewicz et al., 2005 ; 197 séries hydrométriques réparties sur le globe, et Renard, 2006 ; 192 séries hydrométriques sur la France) montrent les difficultés de détection des évolutions globales dans les régimes hydrologiques face au changement climatique.
La première de ces difficultés est liée à la forte variabilité naturelle des phénomènes hydrologiques qui peut masquer la présence de tendances éventuelles. L'application de tests statistiques sur une série trop courte ne permet généralement pas de conclure à des évolutions. La seconde provient des erreurs qui entachent les séries hydrométriques. Celles-ci peuvent être d'ordre métrologique (incertitude des instruments de mesures, des courbes de tarages, des conditions opérationnelles, etc.) ou provenir de l'influence des débits, par la présence d'aménagements hydrauliques par exemple. Comme indiqué par Svensson et al., 2006, il est alors nécessaire de travailler sur un jeu de données exempt de telles influences afin de ne considérer que la variabilité « naturelle » des phénomènes. Enfin, l'interprétation et l'attribution des éventuelles tendances est rendue difficile par la complexité des processus de génération des débits (climatologie, propriétés géomorphologiques des bassins versants, non-linéarité du transfert pluie-débit, etc.). 
L'objectif du travail présenté dans cet article est la détection de tendances dans les longues séries hydrométriques de l'Arc Alpin. Dans cette perspective un jeu de données critiqué et validé couvrant l'ensemble des Alpes a été constitué, et est présenté dans la première partie de cet article. La deuxième partie décrit les indices hydrologiques retenus pour caractériser les basses, moyennes et hautes eaux. La troisième partie présente la méthode statistique de détection de tendance. Les résultats sont détaillés dans la quatrième partie. Enfin la cinquième partie s'attachera à la conclusion de cette étude.

\section{JEU DE DONNÉES}

Collecté dans le cadre du projet INTERREG AdaptAlp (www.adaptalp.org), le jeu de données est bâti à partir des réseaux hydrométriques des six pays partenaires du projet : Allemagne, Autriche, France, Italie, Slovénie et Suisse. Il est constitué de chroniques de débit moyen journalier. Ces données répondent aux critères suivants : (i) les rivières sont géographiquement situées dans les Alpes et une partie au moins de leur régime hydrologique est influencé par la neige, (ii) la longueur de chronique est d'au moins 40 ans, (iii) les stations de jaugeage sont exemptes d'influences majeures.

La sélection des stations est réalisée en deux étapes : premièrement sur la base des méta-données concernant leur qualité et leur exploitation, et deuxièmement au moyen d'une analyse statistique préliminaire de détection de tendances et de ruptures sur les principaux indices hydrologiques (décrits dans la partie III). Cette analyse permet de mettre en évidence les stations qui présentent des variations suspectées non naturelles, typiquement lorsque les changements sont radicaux, généralisés sur un grand nombre d'indices hydrologiques, et concentrés pendant la même période temporelle. Ces stations sont alors passées en revue avec leur gestionnaire afin d'expliquer ces variations et de confirmer leur qualité. Les principaux problèmes rencontrés sont listés dans le Tableau 1.

Le jeu de données final contient 177 séries de mesures pour un total de 119 rivières. Alors que les réseaux de mesure hydrométriques se sont généralisés à partir des années 1950 dans la plupart des pays Européens, la période d'étude offrant la meilleure disponibilité de données est 1961-2005. Entre 126 et 140 stations sont analysées en fonction du type de régime étudié : basses, moyennes, et hautes eaux.

Les surfaces de bassins versants varient de moins de $1 \mathrm{~km}^{2}$ à $15380 \mathrm{~km}^{2}$. Les altitudes des stations de mesure sont comprises entre $79 \mathrm{~m}$ et $1995 \mathrm{~m}$, alors que la plage de l'altitude médiane des bassins versants varie de $368 \mathrm{~m}$ à 2972 m (Figure 1). Cette large diversité physiographique de bassins versants conduit à un large spectre de régimes hydrologiques. La classification des stations par type de régime est réalisée selon la répartition de leurs débits mensuels interannuels (Figure 2), en utilisant la méthode de classification de Kohonen [Wehrens et Buydens, 2007]. Neuf types de régime sont présents dans notre jeu de données, les régimes dits purs : glaciaire, nivo-glaciaire, et nival, puis les régimes appelés nival de transition (subdivisé en deux sous catégories), les régimes « plats » d'influences hydrologiques multiples appelés régimes complexes (subdivisés en deux sous catégories), et enfin les régimes dits mixtes soumis aux influences conjuguées (en proportions variables) de précipitations liquides et solides : nivo-pluvial et pluvio-nival [Pardé, 1955].

\section{INDICES HYDROLOGIQUES}

Toutes les rivières étudiées sont influencées par la fonte, et présentent donc une période de hautes eaux au printemps, ainsi qu'une période hivernale de basses eaux. Les stations qui sont de type mixte sont caractérisées par deux périodes de hautes eaux : fonte printanière et pluies automnales, et deux périodes de basses eaux en été et en hiver. Le calcul des indices hydrologiques s'effectue sur des saisons hydrologiques de six ou douze mois, centrées sur les périodes d'intérêts, le découpage des saisons est fixé selon le type de régime :

- pour les régimes fortement influencés par la fonte nivale (glaciaire, nivo-glaciaire, nival et nival de transition) on distingue une saison centrée sur les hautes eaux, de février à janvier et une saison centrée sur les basses eaux, de juin à mai

- pour les régimes complexes une saison encadrant les hautes eaux, de janvier à décembre et les basses eaux, de mai à avril

- pour les régimes mixtes deux saisons encadrant les hautes eaux, de janvier à juillet et d'août à décembre et deux saisons encadrant les basses eaux, de mai à octobre et de novembre à avril

L'étiage est considéré comme la période pendant laquelle le débit est inférieur à un seuil donné [Fleig et al., 2006]. Dans cette étude ce seuil est fixé comme le quantile à $15 \%$ de la courbe des débits classés, ce qui permet d'obtenir en moyenne un épisode sous seuil par saison. L'intensité des étiages est décrite par trois indices par saison : le déficit de volume, le débit minimum, et la durée de l'étiage. Le déficit de volume correspond au volume manquant par rapport au seuil (quantile à $15 \%$ ). La durée de l'étiage est prise comme le nombre total de jours sous le seuil pendant la saison. La saisonnalité des étiages est caractérisée par trois indices marquant la date de début, centre et fin de l'étiage, qui correspondent aux dates auxquelles le déficit de volume atteint $10 \%, 50 \%$ et $90 \%$ du déficit de volume saisonnier total.

En ce qui concerne les indices de hautes eaux, l'étude porte spécifiquement sur les phénomènes de fonte. Or, à l'échelle journalière, les hydrogrammes peuvent présenter de brusques variations liées à des épisodes pluvieux, qui se superposent à l'onde de fonte. Un algorithme de filtrage a donc été utilisé pour lisser ces brusques variations journalières. L'algorithme proposé par Tallaksen et Van Lanen [2004] a été utilisé dans cette étude. Cet algorithme est généralement utilisé pour extraire le débit de base d'une série hydrométrique; en l'appliquant à des séries à forte influence nivale, on obtient une série où les variations rapides de débit sont lissées, que l'on appellera par la suite « débit de fonte ». L'intensité de la fonte est traduite par le volume total écoulé, et la valeur maximale du débit de fonte. Trois indices de saisonnalité sont également calculés à partir $\mathrm{du}$ débit de fonte, ils correspondent aux dates auxquelles le volume écoulé atteint $10 \%, 50 \%$ et $90 \%$ du volume total, caractérisant le début, centre et fin de l'onde de fonte [Stewart et al., 2005].

Enfin le module (débit moyen annuel), et l'index du débit de fonte (rapport entre le volume du débit de fonte et le volume total écoulé noté BFI) permettent de qualifier le régime des moyennes eaux.

\section{MÉTHODE STATISTIQUE}

L'analyse statistique porte sur la stationnarité des indices hydrologiques précédemment décrits. Les tests sont 
effectués localement pour chaque station. Renard (2006), Lang et al. (2006) et Renard et al. (2006) ont proposé une méthode de sélection du test le plus adéquat selon l'indice étudié et les propriétés des valeurs de l'échantillon. Dans notre cas, les tests non-paramétriques sont préférés car ils ne supposent pas de distribution particulière pour l'indice étudié. Dans cette configuration le test de Mann-Kendall [Kendall, 1975] est utilisé pour la détection de tendances. Le test de Mann-Kendall suppose que les données soient indépendantes, néanmoins cette hypothèse peut s'avérer abusive pour certains indices et ainsi conduire à la détection de tendances inexistantes par le test. Pour remédier à ce problème, Hamed et Rao [1998] ont proposé une correction au test de Mann-Kendall afin de tenir compte de l'autocorrélation dans les séries dans le cas de dépendances dites à « courte portée ». Notons l'existence d'un débat actuellement dans les communautés hydrologiques et climatiques, sur l'existence éventuelle de dépendances dites « à longue portée » dans les séries d'observations hydro-climatiques [Hamed, 2008 ; Koutsoyiannis, 2003 ; Rust et al., 2008]. Cet aspect n'a pas été étudié dans ce travail, nous avons fait l'hypothèse que l'autocorrélation éventuelle affectant les séries d'indices est à « courte portée ».

Les tests locaux sont complétés par une analyse de la significativité régionale. Elle permet de connaître le risque d'erreur à une échelle plus large, c'est-à-dire de connaître le nombre de changements significatifs à partir duquel on peut considérer qu'ils ne sont pas tous dus au simple hasard [Lang et Renard, 2007 ; Renard et al., 2008]. Insistons sur le fait qu'il ne s'agit pas ici d'évaluer la cohérence régionale de changements, mais bel et bien de vérifier que les résultats locaux sont significatifs. En particulier des tendances en sens contraires peuvent très bien être observées au sein de la même région, nous ne nous intéressons ici qu'au nombre de résultats significatifs par signe de tendance (à la hausse ou à la baisse). La méthode utilisée est celle proposée par Douglas et al. [2000] et repose sur une procédure de simulation tenant compte de la dépendance spatiale des données observées.

\section{RÉSULTATS}

Cette partie présente l'ensemble des résultats locaux des tests de détection de tendance au risque de $10 \%$ (risque de première espèce). Ne sont mentionnés dans cette partie que les résultats qui sont régionalement significatifs. Nous indiquons le nombre de stations évoluant de la même manière par rapport au nombre total de stations présentes dans le jeu de données, ou bien de manière spécifique par type de régime hydrologique.

\section{V.1. Etiages hivernaux}

De manière globale la sévérité des étiages hivernaux est en baisse (voir Figure 3) : on observe une diminution significative du déficit de volume (a) et de la durée d'étiage (c) pour $25 \%$ et $26 \%$ des stations, ainsi qu'une augmentation significative du débit minimum (b) pour $25 \%$ des cas. Les résultats concernant la saisonnalité sont peu significatifs à l'échelle globale, excepté pour la date de fin d'étiage (f) qui est plus précoce pour $21 \%$ des stations. Les régimes les plus affectés par la diminution de sévérité sont les régimes glaciaire, nivo-glaciaire et nival. Du point de vue de la saisonnalité les régimes affectés par une fin d'étiage plus précoce sont les régimes glaciaires, nivo-glaciaire, nival, nival-de-transition-1. On observe un décalage des trois indices temporels (début, centre et fin) vers plus de précocité pour les régimes nival-transition-2 et complexe-1, et à l'inverse plus tardif pour les régimes mixtes nivopluvial et pluvio-nival.

\section{V.2. Etiages estivaux}

Ils sont caractéristiques des stations d'influences mixtes nivo-pluviales et pluvio-nivales du piémont des Alpes, et ne concernent donc que 28 stations dans le jeu de données. Concernant la sévérité des étiages estivaux les résultats sont peu significatifs (Figure 4), bien que toutes les tendances significatives détectées pour les indices de déficit de volume (a), du débit minimum (b) et de durée d'étiage (c) aillent dans le sens d'une sévérité accrue, le nombre de changements ne dépasse pas le seuil de significativité régionale. Les résultats montrent un décalage de la saisonnalité plus tôt dans le temps, les dates de début (d), centre (e) et fin (f) sont significativement à la baisse pour $32 \%, 32 \%$ et $29 \%$ des stations (tous régimes mixtes confondus).

\section{V.3. Moyennes eaux}

Les résultats (Figure 5) à grande échelle pour le BFI (a) ne montrent pas de tendance globale : autant de tendances significatives à la hausse qu'à la baisse sont détectées. Néanmoins on peut observer que les stations glaciaires ont un BFI qui augmente significativement (62\% des stations), de même que les stations nivo-glaciaires et nival-de-transition-2 (respectivement $33 \%$ et $21 \%$ ). $\mathrm{Au}$ contraire cet indice est à la baisse pour les régimes nival-de-transition-1, complexe-1 et nivo-pluvial (respectivement $28 \%, 35 \%$ et $22 \%$ ). Les résultats pour le débit moyen annuel (b) présentent très peu de tendances significatives, excepté pour le régime glaciaire où $77 \%$ des stations ont une hausse significative, à l'inverse des régimes mixtes nivo-pluvial et pluvio-nival avec $22 \%$ et $32 \%$ de baisses significatives.

\section{V.4. Hautes eaux}

A l'échelle des Alpes l'intensité des crues nivales ne montre pas de comportement généralisé (Figure 6). Cependant les régimes glaciaires sont particulièrement affectés par une augmentation des crues liées à la fonte : augmentation significative du volume de fonte (a) $(93 \%$ des cas), et du maximum du débit de fonte (b) (47\% des stations). En ce qui concerne les indices de durée (c) et de saisonnalité ( $\mathrm{d}$, e et $\mathrm{f}$ ) les résultats sont plus marqués. Globalement la durée de fonte augmente de manière significative pour $49 \%$ des stations de l'arc alpin, la période de fonte commence également plus tôt dans $49 \%$ des sites, son centre est plus précoce pour $29 \%$ des sites et la fin décalée plus tard pour $16 \%$ des sites. Ces résultats peuvent être précisés par type de régimes. Les régimes glaciaire et pluvio-nival ont leur saison de fonte décalée plus tôt mais sans augmentation de la durée. Les autres régimes ont les dates de début et milieu de fonte plus précoces mais la période de fonte se termine plus tardivement ce qui entraîne une augmentation de la durée. 


\section{TABLEAUX ET FIGURES}

\section{VI.1. Tables}

Tableau 1 : Principaux problèmes de qualité rencontrés, lors de la phase de critique.

\begin{tabular}{|l|c|}
\hline \multicolumn{1}{|c|}{$\begin{array}{c}\text { Problèmes } \\
\text { de qualité rencontrés }\end{array}$} & $\begin{array}{c}\text { Nombre } \\
\text { de stations exclues }\end{array}$ \\
\hline Aménagement hydrauliques amonts & 37 \\
\hline Problèmes métrologique & 17 \\
\hline Problème de gestion & 9 \\
\hline Métadonnées ou qualité inconnue & 36 \\
\hline
\end{tabular}

VI.2. Figures

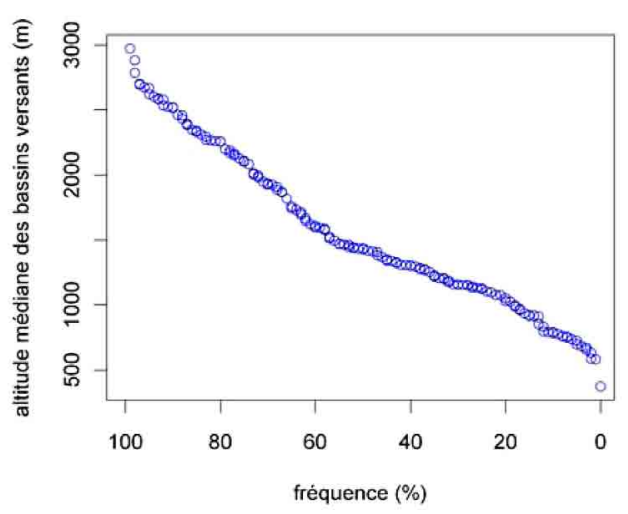

Figure 1 : Répartition de l'altitude médiane des bassins versants.
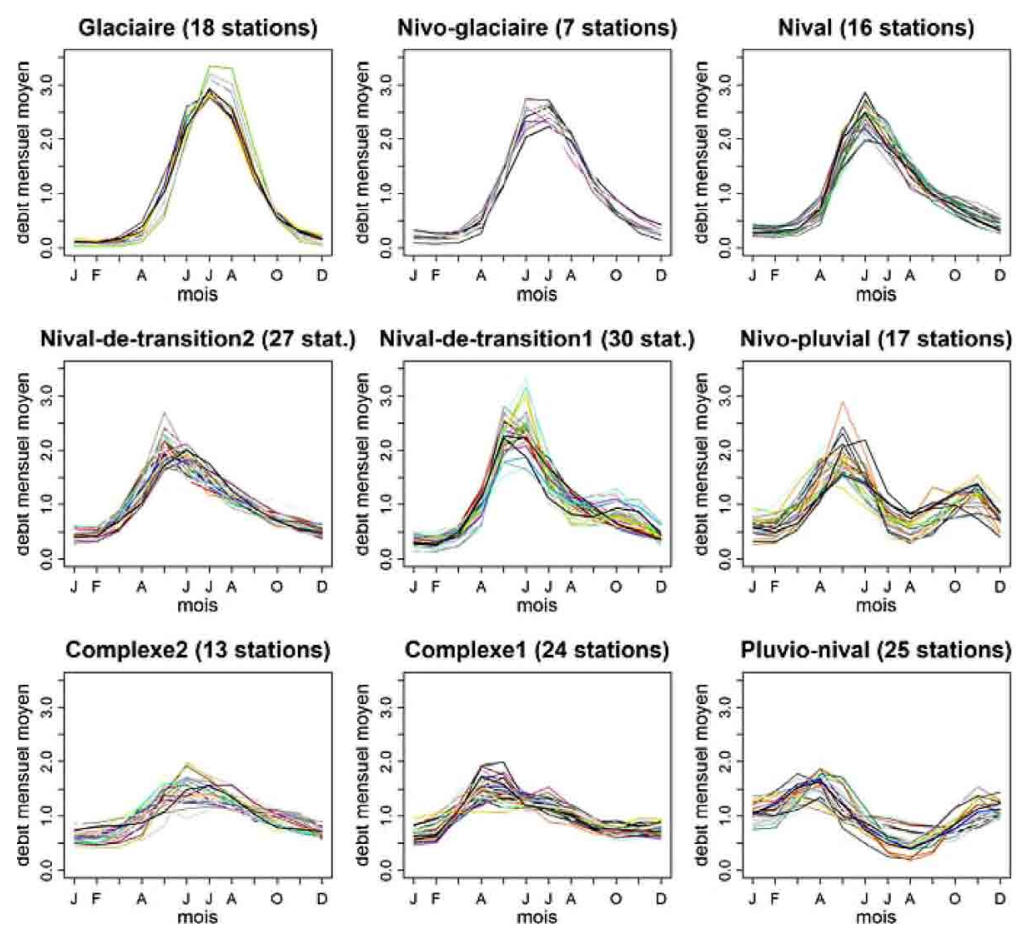

Figure 2 : Régimes hydrologiques.

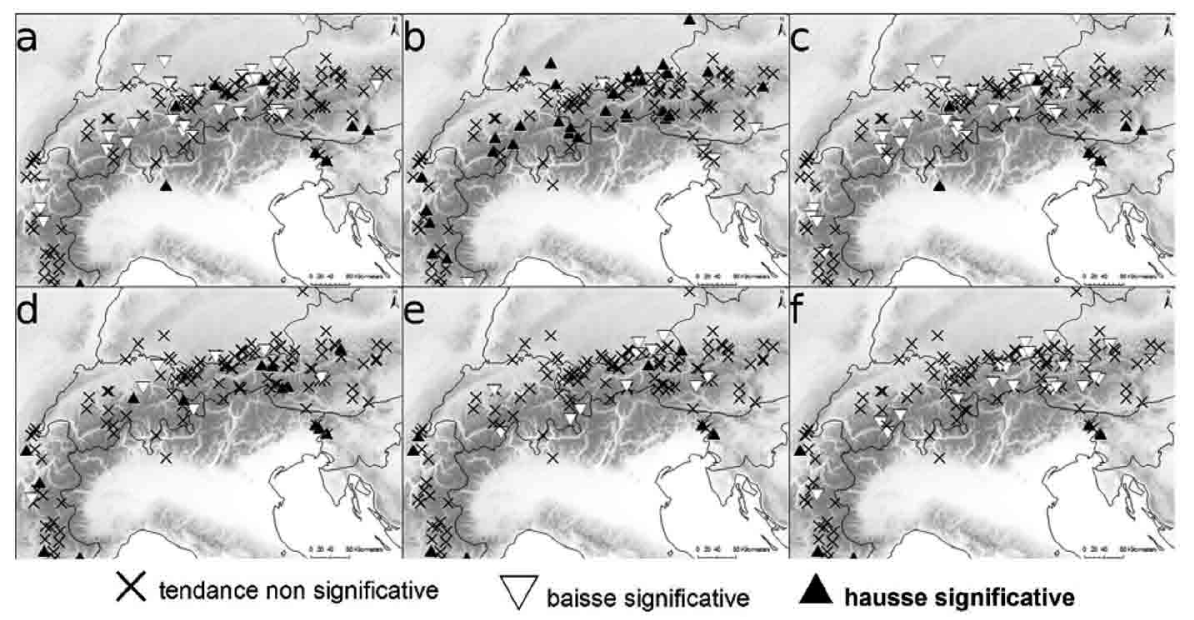

Figure 3 : Carte des résultats locaux pour les étiages hivernaux: (a) déficit de volume total, (b) valeur du débit minimum, (c) durée, (d) date de début, (e) date de centre, (f) date de fin. 


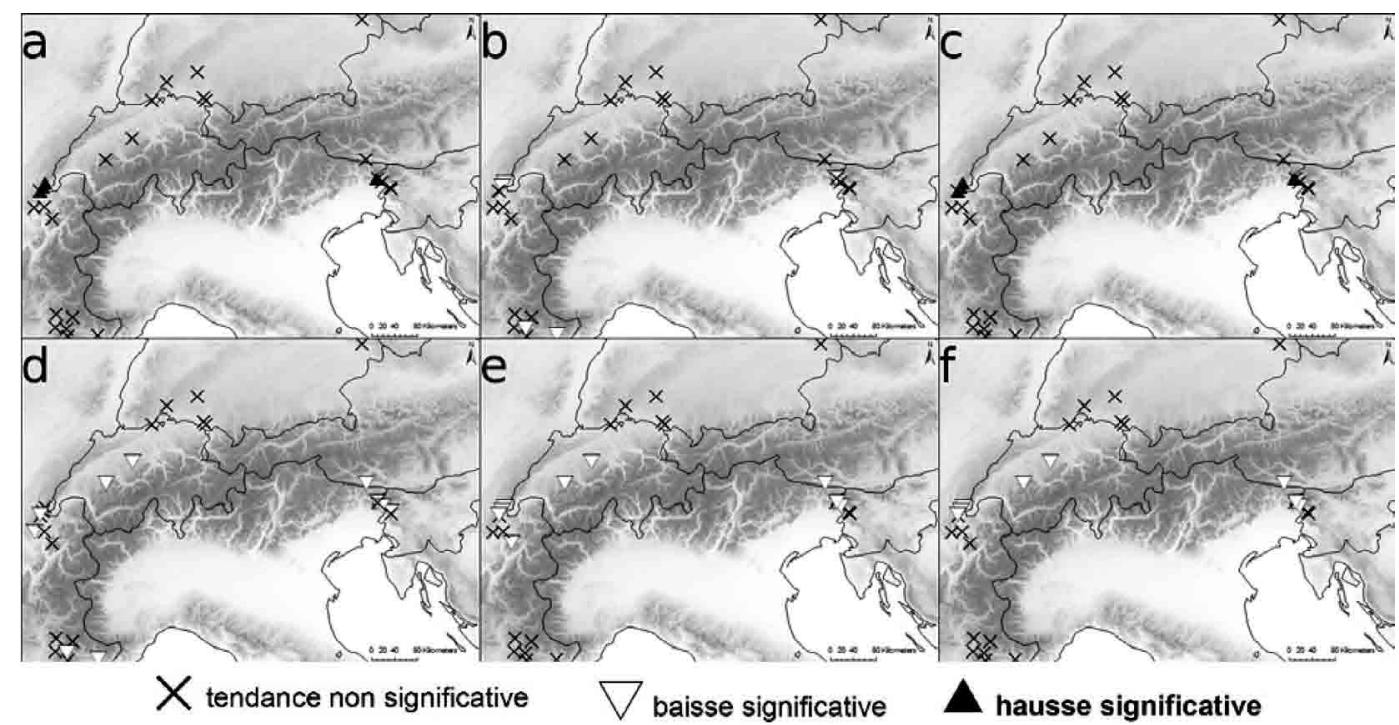

Figure 4 : Carte des résultats locaux pour les étiages estivaux: (a) déficit de volume total, (b) valeur du débit minimum, (c) durée, (d) date de début, (e) date de centre, (f) date de fin.

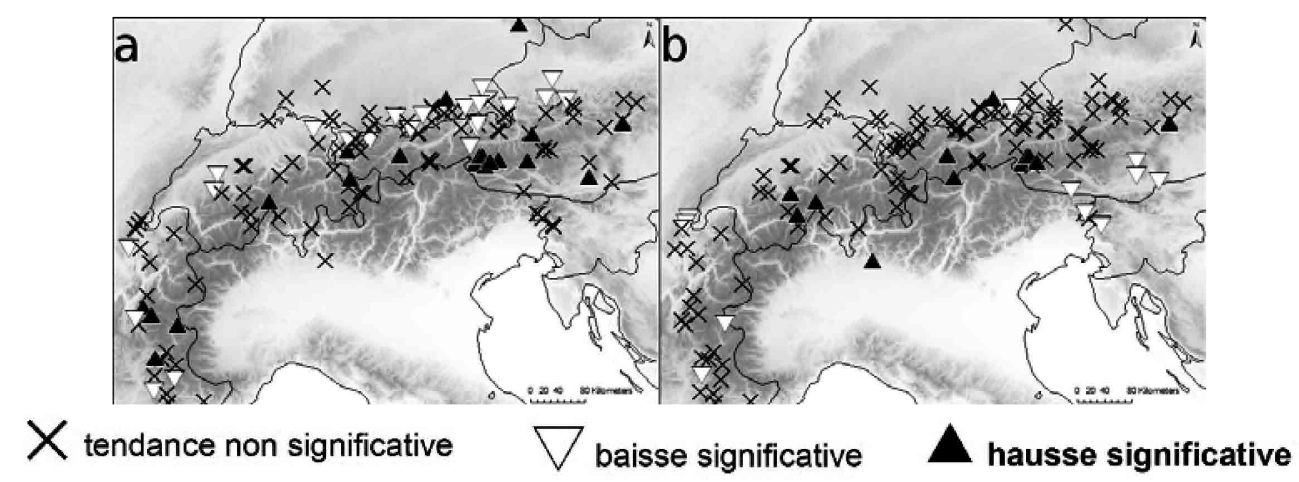

Figure 5 : Carte des résultats locaux pour les moyennes-eaux : (a) le BFI et (b) le débit moyen annuel.

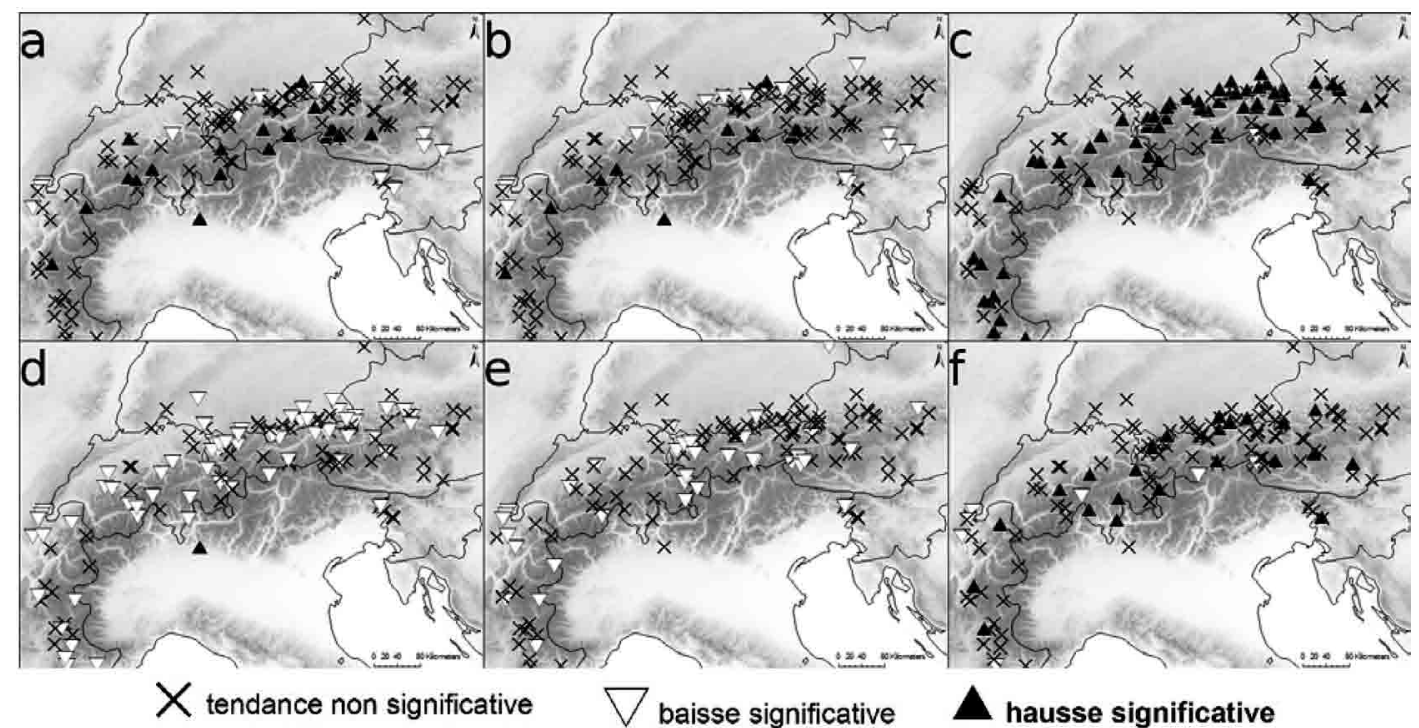

Figure 6 : Carte des résultats locaux pour les crues de fonte: (a) volume total du débit de fonte, (b) maximum du débit de fonte, (c) durée de la fonte, (d) date de début, (e) date de centre, (f) date de fin. 


\section{CONCLUSION}

Cette étude se base sur une méthode éprouvée pour la détection de tendance et largement utilisée dans le domaine de l'hydrologie [Stewart, 2005 ; Birsan et al., 2005 ; Pellicciotti et al., 2010]. Les résultats obtenus pour 177 longues séries hydrométriques montrent des changements globaux à l'échelle des Alpes. Pour les basses eaux on observe une baisse significative de la sévérité des étiages hivernaux (diminution du déficit de volume, augmentation $\mathrm{du}$ débit minimum), ainsi qu'un raccourcissement de la durée d'étiage. Pour les hautes eaux, on peut observer un décalage temporel de la période de fonte (vers une précocité accrue) et un allongement marqué de la durée de fonte $(50 \%$ des sites étudiés). Les changements d'intensité de la fonte nivale sont cantonnés aux régimes d'influence glaciaire pour lesquels les volumes de fonte sont en augmentation. De manière générale les résultats sont très structurés selon les différents types de régimes hydrologiques.

Il est important de garder à l'esprit les limites de la méthode utilisée. Premièrement soulignons la limite de la puissance des tests statistiques : une tendance non significative ne prouve pas l'absence de tendance, mais illustre simplement l'incapacité du test à la détecter. Deuxièmement cette méthode ne permet pas de conclure sur la cohérence régionale des changements détectés. Ce travail est cependant en cours de réalisation et sera décrit dans un prochain article.

Bien que les résultats obtenus puissent être reliés de manière intuitive aux élévations de températures mesurées dans les différentes parties des Alpes, cette étude ne permet pas de démontrer formellement que les évolutions observées sont la conséquence directe et unique du réchauffement anthropique. En effet, il faudrait dans un premier temps dresser un panorama complet des évolutions affectant les variables de forçage du débit, car les évolutions dans les pluies et températures (par exemple) peuvent avoir des effets antagonistes sur les débits. De plus, la complexité de la transformation précipitation-débit rend difficile tout raisonnement intuitif, et rend indispensable le recours à la modélisation hydrologique pour appréhender la réponse attendue des bassins versants à l'évolution des forçages. Notons que ce type d'approche possède des similarités avec les études d'attribution [Bernstein et al., 2007] réalisées par les climatologues pour attribuer une tendance au réchauffement d'origine anthropique.

En dépit de ces limitations, l'excellente cohérence des évolutions observées sur certains indices indique que les rivières Alpines pourraient fournir un terrain d'étude prometteur pour mieux comprendre les relations entre les variabilités climatique et hydrologique

\section{RÉFÉRENCES ET CITATIONS}

Bernstein L., P. Bosch, O. Canziani, Z. Chen, R. Christ, O. Davidson, W. Hare, S. Huq, D. Karoly, V. Kattsov, Z. Kundzewicz, J. Liu, U. Lohmann, M. Manning, T. Matsuno, B. Menne, B. Metz, M. Mirza, N. Nicholls, L. Nurse, R. Pachauri, J. Palutikof, M. Parry, D. Qin, N. Ravindranath, A. Reisinger, J. Ren, K. Riahi, C. Rosenzweig, M. Rusticucci, S. Schneider, Y. Sokona, S. Solomon, P. Stott, R. Stouffer, T. Sugiyama, R. Swart, D. Tirpak, C. Vogel, G. Yohe (2007) Climate Change 2007 : Synthesis Report. GIEC

Birsan M.V., P. Molnar, P. Burlando, M. Pfaundler (2005) Streamflow trends in Switzerland. Journal of Hydrology 314(1-4) 312-329
Douglas E.M., R.M. Vogel, C.N. Kroll (2000) - Trends in floods and low flows in the United States : impact of spatial correlation. Journal of Hydrology 240(1-2) 90-105

Fleig A.K., L.M. Tallaksen, H. Hisdal, S. Demuth (2006) - A global evaluation of streamflow drought characteristics. Hydrology and Earth System Sciences 10 535-552

Hamed K.H.A., R. Rao (1998) - A modified Mann-Kendall trend test for autocorrelated data. Journal of Hydrology 204(1-4) 182-196

Hamed K.H. (2008) - Trend detection in hydrologic data : The Mann-Kendall trend test under the scaling hypothesis. Journal of Hydrology 349(3-4) 350-363

Kendall M.G. (1975) - Rank correlation methods. London : Griffin

Koutsoyiannis D. (2003) - Climate change, the Hurst phenomenon, and hydrological statistics. Hydrological Sciences JournalJournal Des Sciences Hydrologiques 48(1) 3-24

Kundzewicz Z.W., D. Graczyk, T. Maurer, I. Pinskwar, M. Radziejewski, C. Svenssonm. Szwed (2005) - Trend detection in river flow series 1. Annual maximum flow. Hydrological Sciences Journal-Journal Des Sciences Hydrologiques 50(5) 797-810

Lang M., B. Renard, E. Sauquet, P. Bois, A. Dupeyrat, C. Laurent, O. Mestre, H. Niel, L. Neppel, J. Gailhard (2006) - A national study on trends and variations of French floods and droughts. Climate Variability and Change. Hydrological Impacts 308 514-519

Lang M., B. Renard (2007) - Analyse régionale sur les extrêmes en France : détection de changements cohérents et recherche de causalité hydrologique. La Houille Blanche. 6 83-89

Parde M. (1955) - Fleuves et rivières. Paris : Armand Colin 1

Pellicciotti F., A. Bauder, M. Parola (2010) - Effect of glaciers on streamflow trends in the Swiss Alps. Water Resources Research. 46 1-16

Puget J.L., R. Blanchet, J. Salençon, A. Carpentier (2010) Le changement climatique. Académie des sciences. Synthèse des débats sur le climat, 20 sept. $21 \mathrm{p}$

Renard B. (2006) - Détection et prise en compte d'éventuels impacts du changement climatic sur les extrêmes hydrologiques en France. Thèse INP Grenoble, Cemagref Lyon 362p

Renard B., M. Lang, P. Bois, A. Dupeyrat, O. Mestre, H. Niel, J. Gailhard, C. Laurent, L. Neppel, E. Sauquet (2006) Evolution des extrêmes hydrométriques en France à partir de données observées. La Houille Blanche. 6 48-54

Renard B., M. Lang, P. Bois, A. Dupeyrat, O. Mestre, H. Niel, E. Sauquet, C. Prudhomme, S. Parey, E. Paquet, L. Neppel, J. Gailhard (2008) - Regional methods for trend detection : Assessing field significance and regional consistency. Water Resources Research. 44(8) 1-17

Rust H.W., O. Mestre, V.K.C. Venema (2008) - Fewer jumps, less memory : Homogenized temperature records and long memory. Journal of Geophysical Research-Atmospheres. 113(D19)

Stewart I.T., D.R. Cayan, M.D. Dettinger (2005) - Changes toward earlier streamflow timing across western North America. Journal of Climate. 18(8) 1136-1155

Svensson C., J. Hannaford, Z.W. Kundzewicz, T.J. Marsh. (2006) - Trends in river floods : why is there no clear signal in observations? in Frontiers in Flood Research. Wallingford: Int Assoc Hydrological Sciences

Tallaksen L.M., H.A.J. Van Lanen (2004) - Hydrological Drought : processes and estimation methods for streamflow and groundwater. Developments in Water Science, ed. L.M. Tallaksen and H.A.J. Van Lanen : Elsevier

Viviroli D., R. Weingartner (2004) - The hydrological significance of mountains : from regional to global scale. Hydrology and Earth System Sciences 8(6) 1016-1029

Wehrens R., L.M.C. Buydens (2007) - Self- and Super-organizing maps in R : the Kohonen Package. Journal of Statistical Software. 21 1-19 